\title{
Research on Different Emotional Characteristics of Driver
}

\author{
Anqi $\mathrm{Ji}^{1, \mathrm{a}^{*}}$, and Jihui $\mathrm{Ma}^{2}$, b \\ ${ }^{1}$ MOE Key Laboratory for Urban Transportation Complex Systems Theory and Technology, School \\ of Traffic and Transportation, Beijing Jiaotong University, China \\ ${ }^{2}$ Institute of Systems Engineering and Control, School of Traffic and Transportation, Beijing \\ Jiaotong University, China \\ a16120762@bjtu.edu.cn, bjhma@bjtu.edu.cn
}

Keywords: Road traffic safety; Driver emotion; Driver behavior; Physiological signal

\begin{abstract}
With the increasing complexity of the traffic system, the study of driver emotion has become a hot topic. Traffic psychology mentioned that both the driver's character and the emotional changes in the driving process will affect the driver's driving behavior. Through real-time tracking of human physiological signal changes to identify the driver's emotions and analyze their driving intention, and then study the driving behavior. This paper analyzes the ECG, skin signal, EMG, heart rate and other physiological signals and pedal force of drivers under different emotions. The Bayesian network model is used to effectively identify the emotional characteristics of the driver in the process of driving. This research is of great significance to intelligent vehicle development and traffic safety management and intelligent city construction.
\end{abstract}

\section{Introduction}

The car- road - environment affects the road traffic safety by influencing the driver's emotions. Therefore, the driver of different emotional characteristics of the information collection and processing, to identify different emotional characteristics, and will enable the car safety driving system can be more intelligent on the implementation of human protection, improve the protection accuracy, and effectively prevent the occurrence of traffic accidents [1,2].

The main research work is as follows: designing mental questionnaire, carrying out driving simulation experiment, building a mathematical model and combing with the experimental data, analysis of the driver's driving intention.

\section{Simulated Driving Experiment}

Through this experiment, the physiological signals in the course of the pilot experiment are extracted, and the emotional characteristics data of the driver in the vehicle running are obtained. The driver's emotion and intention are analyzed to explore the influence of emotion on the intention.

Through the study found that ECG signal waveform, including P wave, QRS wave group and T wave $[3,4,5]$. The basic principle of muscle electrical sensor measurements is to amplify and measure these tiny electrical pulses, the specific details shown in Fig. 1.

Various experimental equipment and the corresponding model as shown in Table 1. 
Table 1 Equipment

\begin{tabular}{|c|c|c|}
\hline Number & Equipment & model \\
\hline 1 & Pedal force control & WTC-1 \\
\hline 2 & Driver's physiological signal acquisition telemetry monitoring system & BIOHARNESS \\
\hline 3 & Plug-in multi-function navigation recorder & Columbus_V-900 \\
\hline 4 & Steering parameter tester & SG299-2D \\
\hline 5 & Non-contact multi-function speed meter \\
\hline 6 & PsyLAB engineering test system & \\
\hline 7 & Car driving simulation system & \\
\hline
\end{tabular}
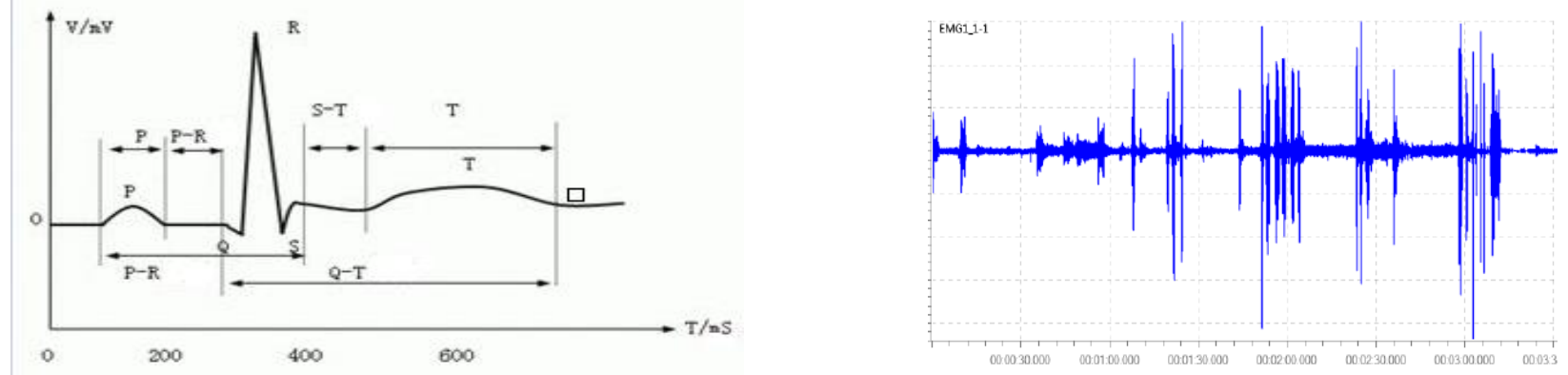

Figure $1 \quad$ ECG and EMG signal waveform

Respondents' breathing intensity and respiration rate were measured by testing the belt's tensile strength [6]. EDA is generated by the current flow in the skin generated by the potential difference, the potential difference with the subject's visual, auditory, tactile and other stimuli and emotional evolution and change, the specific details shown in Fig. 2.
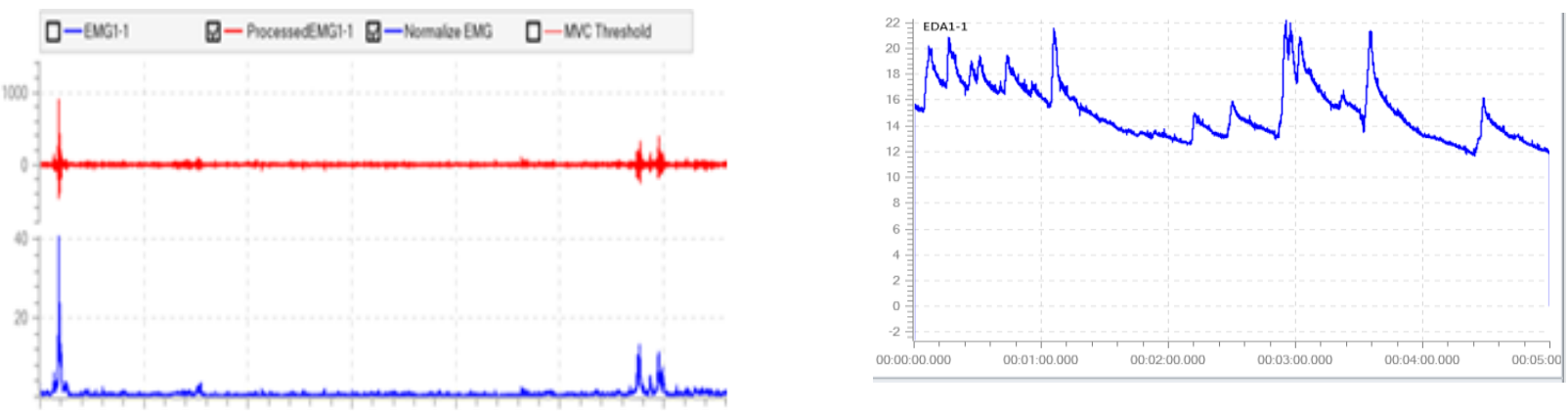

Figure 2 RSP and EDA signal waveform

\section{Construction of Bayesian Model}

The driver's character can be divided into adventure, cautious and conservative [7]. According to the driver's personality and the external environment changes as the driver's emotional characteristics and driving behavior of the interference factors, to build the emotional state of Bayesian network.

In Fig. 3, assuming $\mathrm{E}=\left(e_{1}, e_{2}, e_{3} \ldots e_{i} \ldots e_{n}\right)$ indicates the emotional state that may be experienced during driving, $\mathrm{C}=\left(c_{1}, c_{2}, c_{3} \ldots c_{p} \ldots c_{l}\right)$ indicates the personality of the driver, $\mathrm{J}=\left(j_{1}, j_{2}, j_{3} \ldots j_{q} \ldots j_{m}\right)$ indicates traffic information. 


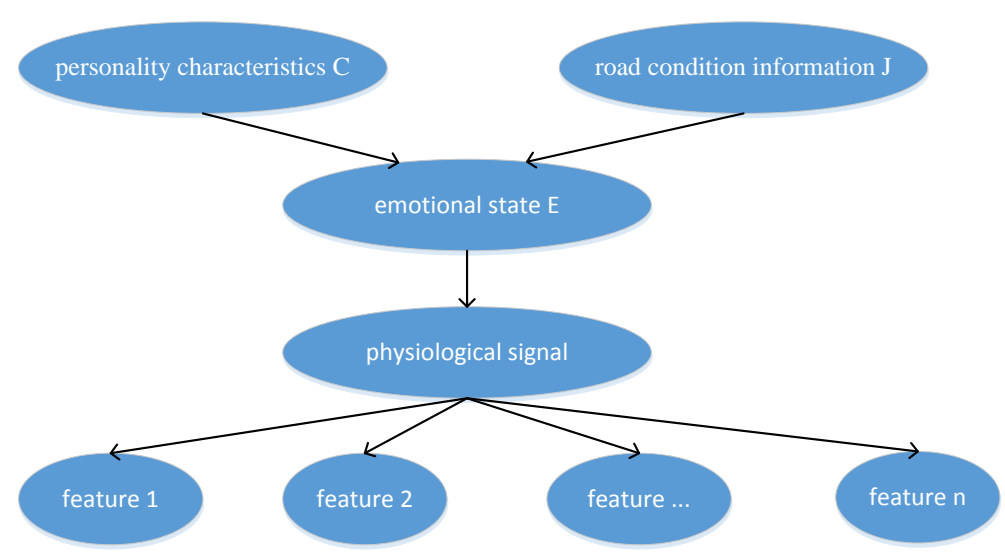

Figure 3 the emotional state of Bayesian network

According to the special relationship between the models $\mathrm{E}, \mathrm{C}$ and $\mathrm{J}$, can be set $p\left(e_{i} / c_{p}, j_{q}\right)$ (Speak with $p\left(e_{i}\right)$,If the probability of occurrence after the distribution in the absence of special instructions are in the case of $\left.\left(c_{p}, j_{q}\right)\right)$. The probability distribution of the eigenvalues under known emotional conditions can be obtained by experiments: $p\left(t_{k}, e_{i}\right)$.

The joint probability distribution between the eigenvalue and the emotional state of the physiological signal can be obtained by the principle of joint probability distribution, showed in Eq.1.

$$
p\left(t_{k}, e_{i}\right)=p\left(t_{k} / e_{i}\right) * p\left(e_{i}\right)
$$

In formula, $t_{k}$ represents the kth characteristic of the physiological electrical signal.

The summation of the sum of all the numbers can give the probability distribution of the kth characteristic of the physiological signal in Eq.2.

$$
p\left(t_{k}\right)=\sum_{i=1}^{n} p\left(t_{k}, e_{i}\right)=\sum_{i=1}^{n} p\left(t_{k} / e_{i}\right) * p\left(e_{i}\right)
$$

According to the theory of conditional probability, we can get the probability distribution of emotional state under the condition of known feature distribution, that is, the posterior probability distribution of emotional state in Eq.3.

$$
p\left(e_{i}\right)=p\left(e_{i} / t_{k}\right)=\frac{p\left(t_{k}, e_{i}\right)}{p\left(t_{k}\right)}
$$

Considering the better variance of the Welch method, the Welch method is used to estimate the power spectrum.

The Welch method allows each segment to be partially overlapped. The data window $d(n)$ of the segment may be a rectangular window and Henning window. Let the power spectrum of the i-th segment be

$$
\begin{gathered}
p_{\text {PER }}(f) \\
p_{\text {PER }}(f)=\frac{1}{M U}\left|\sum_{i=0}^{M-1} x_{N}^{i}(n) d(n) e^{-j 2 \pi \mathrm{fn}}\right|^{2}
\end{gathered}
$$

In formula, $U=\frac{1}{M} \sum_{n=0}^{M-1} d^{2}(n)$ then, The average power spectrum is given by the following
equation:

$$
\tilde{p}_{P E R}(f)=\frac{1}{L} \sum_{i=1}^{L} \hat{p}_{P E R}^{\wedge i}(f)=\frac{1}{L M U} \sum_{i=1}^{L}\left|\sum_{n=0}^{M-1} X_{N}^{i}(n) d(n) e^{-j \pi 2 f n}\right|^{2}
$$


Because of the individual differences between each individual, the physiological and electrical signals are also very different [8]. In order to achieve the purpose of normalization, the relative power spectrum values of the three bands in $0.5-20 \mathrm{~Hz}$ are used as candidate feature information.

The eigenvalues of the relative power spectra of the four bands in different states are calculated according to the above equation. Since the difference between the mean values of the two eigenvalues is larger and the variance of the eigenvalues of each state is smaller, the two states are more easily distinguishable, so the principle of feature extraction is proposed Eq.6.

$$
f_{i j}=\frac{F_{i j}}{S_{i}+S_{j}}=\frac{\left(\operatorname{mean}\left(t^{i}\right)-\operatorname{mean}\left(t^{j}\right)\right)^{2}}{\left(t^{i}-\operatorname{mean}\left(t^{j}\right)\right)^{2}+\left(t^{j}-\operatorname{mean}\left(t^{j}\right)\right)^{2}}, i \neq j
$$

In formula, $t_{i}$ denotes the eigenvalue of the $\mathrm{i}$-th state, $\mathrm{F}_{i j}$ denotes the degree of difference between the corresponding eigenvalues of the two states, and $S_{i}$ denotes the $t_{i}$ mean square error, and the feature that maximizes $\mathrm{f}_{i j}$ is the desired feature.

\section{Model Validation}

The driver accelerates the accelerator pedal and the brake pedal and the speed of the vehicle can be directly from the simulation system in the data acquisition system real-time output $[9,10]$, the vehicle acceleration can be calculated according to the speed by the Eq.7.

$$
a_{t}=\frac{V_{t}-V_{t-1}}{3.6}
$$

In formula, $a_{t}$ is the acceleration of the $\mathrm{t}$-th vehicle $\left(\mathrm{m} / \mathrm{s}^{2}\right), v_{t}$ is the speed of the $\mathrm{t}$-th vehicle $(\mathrm{km} / \mathrm{h})$.

In this part, we use the PsyLAB engineering experiment system to measure the two sets of data of happy and angry emotions, and randomly select 60 data to train them. According to the Bayesian network model reasoning, we obtain the characteristic emotion probability distribution Law, and thus the final emotional definition criteria: While, $28<\mathrm{t} \leq 90$, decided to be happy; $90<\mathrm{t} \leq 120$, Judged to be angry

The results show that the accuracy of the model is $77.02 \%$. In order to ensure the correctness of the conclusion, 400 sets of data are selected and analyzed. The results show that the accuracy of the test is $76.25 \%$.

\section{Summary}

The content of this paper is the acquisition and processing of data under different emotional characteristics, the use of the cutting edge of the world's leading equipment for data collection, through the construction of Bayesian network model for data analysis and processing [11]. In the analysis, the relative power spectrum of different ECG signals is used as the characteristic to study $[12,13]$. The results show that the method has high recognition degree.

\section{References}

[1] Christine Mohn, Heike Argstatter and Friedrich Wilhelm Wilker: Psychology of Music, Vol. 39 (2014) No.4, p.503.

[2] T.Y. Hu, X.F. Xie and J. Li: Driving Transportation Research Part F, Vol.6 (2013) No.6, p.29.

[3] Amata Hideomi, Miyajima Chiyomi, Nishino Takanori and Kitaoka Norihide: 8th International Conference on Intelligent Transportation Systems (Las Vegas, Nevada, 2015) Vol.1, p.747.

[4] Ko J, Guensler R and Hunter M: Driving Transportion Research Part F, Vol.13 (2013) No.1, p. 21. 
[5] Suresh Pandian, Sharad Gokhale and Aloke Kumar Ghoshal: Transportation Research Part D, Vol.14 (2012) No.4, p. 180.

[6] Hakuya M, Pongsathorn R and Macao N: International Joint Conference (Bilbao, Spain, June 25-27, 2014) Vol.1, p.534.

[7] Abdu Rotem, Shinar David and Meiran Nachshon: Transportation Research Part F, Vol.15 (2012) No.5, p.575.

[8] Brundell-Freij K and Ericsson E. Transportion Research Part D, Vol.10 (2015) No.3, p. 213.

[9] Gunnarsson J, Svensson L and Bengtssont E. IEEE Transactions on Multimedia, Vol.13 (2011) No.5, p. 1180.

[10] Karin Brundell-Freij and Eva Ericsson: Transportation Research Part D, Vol10 (2015) No.3, p.213.

[11] Alberto Megias, Antonio Maldonado, Antonio Candido and Andres Catena: Accident Analysis and Prevention: Vol.43 (2011) No 3, p. 813.

[12] YukiT, Takashi O and Koichiro Y: Proceedings of the 2007 IEEE Symposium on Foundations of Computational intelligence (Honolulu, Hawaii, USA, 2013) Vol.12, p. 289.

[13]Changiz Mohiyeddini, Regina Pauli and Stephanie Bauer. Psychology of Sport and Exercise, Vol.10 (2013) No.2, p. 226. 